\title{
Archéopages
}

Archéopages

Archéologie et société

Hors-série 1 | 2008

Constructions de l'archéologie

\section{La nostalgie de l'avenir : souvenir de médiévie intérieure}

Joëlle Burnouf

\section{OpenEdition}

1 Journals

Édition électronique

URL : https://journals.openedition.org/archeopages/873

DOI : 10.4000/archeopages.873

ISSN : 2269-9872

Éditeur

INRAP - Institut national de recherches archéologiques préventives

Édition imprimée

Date de publication : 1 février 2008

Pagination : 105-106

ISSN : 1622-8545

\section{Référence électronique}

Joëlle Burnouf, «La nostalgie de l'avenir : souvenir de médiévie intérieure », Archéopages [En ligne],

Hors-série 1 | 2008, mis en ligne le 01 février 2008, consulté le 27 février 2023. URL : http://

journals.openedition.org/archeopages/873; DOI : https://doi.org/10.4000/archeopages.873

Tous droits réservés 


\section{La nostalgie de l'avenir: souvenir de médiévie intérieure}

Joëlle Burnouf

Université Paris I Panthéon-Sorbonne,

UMR 7041 ArScAn «Archéologies environnementales»

S uivant le conseil d'André Leroi-Gourhan - avant de taper frénétiquement sur le clavier de mon ordinateur et parce que j'allais faire quelque chose d'important-, j'ai reculé et allumé une cigarette.

Difficile de prendre de la distance: je me trouvais mise en demeure d'écrire «un texte», alors que le point commun à tous les archéologues est d'être spécialistes des documents «aphones». J'aurais pu prendre cet angle d'attaque pour dire «Cela va sans dire», point.

Examiné sous cet aspect, le métier est éclairé d'un jour nouveau. Il fut un temps où l'on qualifiait notre documentation de «muette», mais, comme nous la faisions parler, le paradoxe s'étalait aux yeux de tous. Ils avaient bien de la chance, les spécialistes des périodes d'avant l'écriture, de n'avoir à traiter que des documents «aphones», car la conquête de notre identité de «médiévistes sédimentaires» fut des plus difficiles en raison de l'existence de documents «bavards» : les sources écrites. Pendant trente ans, entre sources aphones et textes bavards, ce fut un impossible dialogue. Ce temps est révolu, mais il reste du chemin à parcourir. Et la conquête de cette identité doit beaucoup au développement de l'archéologie préventive.

«Il était une fois l'archéologie préventive». Lancée au milieu des années 1970, l'archéologie préventive fut d'abord une aventure intellectuelle terriblement physique où il fallait tout inventer, ici et maintenant, là et ailleurs: de la négociation des dossiers aux stratégies de fouille, les infatigables terrassiers de l'histoire vibrillonnaient pour convaincre. C'était, en ce temps-là, une archéologie militante.

Certaines rencontres se firent, qui étaient improbables, les vallées de l'Aisne et du Rhône (ou du Rhin suivant l'endroit où je fouillais) n'appartenant pas au même bassin versant. Heureusement, les «tribus» ont leur grand-messe où elles communient dans le culte de la période! Chaque année, les clans et les lignages se livrent à des ambulations rituelles pour rejoindre un point de l'Hexagone où sont présentés les fleurons des découvertes. Tout le monde se connaît, tout le monde identifie les papes, les cardinaux, les thuriféraires, les hérétiques. Mais, dans la grande «famille», on est entre soi. Ce sont les hasards de l'archéologie préventive qui m’ont fait participer à l'une de ces manifestations dans le grand amphi de l'Institut d'histoire de l'art et d'archéologie à Paris, la Mecque de l'archéologie théorique depuis les années 1970. Et c'est là, au milieu des années 1980, que je rencontrai pour la première fois la «tribu» parisienne des protohistoriens, tribu dont la pensée s'appuyait sur la «new archaeology» qui avait gagné la province, laquelle tentait, à son tour, de faire évoluer l'archéologie en s'inspirant des livres fondateurs de ces années. Et quelle ne fut pas ma surprise de constater que cette mythique tribu était composée de membres jeunes et séduisants, dont Jean-Paul Demoule, et non de vieux archéologues chenus aux cheveux blancs!

Dix ans de luttes! Puis, en 1990, je fus invitée à entrer dans le comité de rédaction de la revue Les Nouvelles de l'archéologie, sis au sein de la Fondation de la Maison des sciences de l'homme à Paris.

Le comité de rédaction des Nouvelles était, en ce temps-là, un endroit improbable que je n'ai jamais vu ailleurs, et qui n'a aujourd'hui aucun parallèle. Il était large, et nous étions nombreux à venir de partout le samedi matin - une matinée qui durait jusqu'à ce que la Maison fermepour y faire, sans doute, le travail d'un comité de rédaction, mais surtout pour y débattre librement sur la discipline, son présent, son avenir. Les ordres du jour étaient inépuisables et inépuisés. Les «samedis des Nouvelles» furent un formidable espace de liberté et de débat, la revue un lieu tonique où l'on pouvait exprimer les idées nouvelles. Il était aussi peuplé des grands noms de l'archéologie en gestation, des grandes plumes qui racontaient une autre histoire, et de voix aussi qui faisaient entendre des points de vue prospectifs sur nos métiers. Rétrospectivement, l'aventure de ces années était «politique».

Il était demandé aux membres du comité de rédaction de proposer et cornaquer un «dossier» des Nouvelles. Lectrice de la revue depuis le numéro o (un «collector»!), j'avais quelques idées qui me tenaient à cœur, et surtout une. Dans une vie antérieure, lorsque j'officiais en Lorraine comme chef du service régional de l'Archéologie, j'avais eu l'idée d'organiser pour le bicentenaire de la Révolution - un congrès européen au Moulin de Valmy (pour le symbole de la «levée en masse»), sur «Archéologie et idéologie». Des personnes raisonnables en haut lieu surent me faire comprendre «qu'à l'homme de la rue j'avais des comptes à rendre» et qu'il était urgent d'attendre.

Puis vinrent les années 1990 et la montée électorale du Front national: les questions écrites posées au ministre de la Culture par les mêmes nous renvoyaient à nos luttes. Ils étaient les premiers à interroger sur l'érosion du patrimoine enfoui avec des arguments qui n'avaient de cesse d'inquiéter. Mon projet fut donc repris un samedi matin: ce fut le dossier "Archéologie et identité», réalisé en collaboration avec Jean-Paul Demoule et Armelle Bonis. Je devais le poursuivre, jusqu'en 2003, sous une forme pluridisciplinaire faisant intervenir des ethnologues et des historiens, dans le cadre d'un programme du ministère de la Recherche, dirigé par Anick Coudart. 
Les années CNRA: «Nous l'avons tant aimée, l'archéologie préventive». Dans ces mêmes années, marquées par les deux premiers épisodes de «la guerre des truelles», cette lutte obstinée pour bâtir une archéologie de service public, obtenir une loi sur l'archéologie et construire un établissement public, le CsRA (Conseil supérieur de la recherche archéologique) - modèle ancien-, venait d'imploser en séance plénière de ce qu'il est coutume d'appeler, dans nos milieux, «un colloque de restitution». Un CNRA (Conseil national de la recherche archéologique) allait être mis en place; le bras de fer pour les places des élus comme des nommés fut un grand moment! D'autant que nous n'y allions pas pour nous tourner les pouces mais pour mettre en œuvre «la réforme» et fonder l'établissement pas question d'une autre forme que celle d'un établissement «public». Les séances étaient longues et animées: nous étions dans l'urgence d'agir par nostalgie de l'avenir. Parfaitement conscients de notre nouveau statut de «papys and mamies boomers», le demi-siècle nous avait rattrapés. Nous cherchions des jeunes (enfin... des plus jeunes que nous!) et des femmes (la parité autant que faire se pouvait). Chacun, dans nos réseaux, nos «hordes», nous relancions sans relâche les nouvelles générations. Dans ces débats et combats, nous avions un point commun: de solides ennemis. Que faire? Aller de l'avant, continuer à inventer «l'archéologie de demain», expliquer et inlassablement expliquer pour convaincre.

Les invisibles de l'histoire. Si nous avions eu un slogan, c'eût pu être: "Nous sommes tous des historiens sédimentaires en lutte contre l'amnésie.» Sachant que l'amnésie a toujours eu un fort coût social, la tâche était rude car le quotidien de l'archéologie préventive métropolitaine et d'outre-mer n'arborait pas les atours rutilants de Néfertiti. Nous besognions des documents aphones qui permettaient de faire parler les «invisibles de l'histoire». C'était généralement aride et plutôt abstrait. Bref, nous étions en quelque sorte des décodeurs. Il fallait donner à voir et à montrer comment ces «invisibles de l'histoire» avaient construit le présent.

Sur le terrain, les archéologues avaient la pratique quotidienne du dialogue avec les aménageurs, les décideurs et les élus. Ils avaient construit des argumentaires qui leur permettaient de faire comprendre leurs travaux et leurs résultats. Les grands espaces explorés changeaient peu à peu l'intelligence des réseaux, des groupes sociaux de leurs relations...

Les archéologues avaient construit la «géographicité» de l'archéologie, ils démontraient, chaque jour, les capacités d'aménagement du territoire des sociétés du passé. Ils donnaient à voir les héritages résilients sans cesse remodelés, travaillés, transformés et, jusqu'à leurs travaux, occultés - car, sans médiateurs pour les comprendre, il n'était pas d'écriture de l'histoire.
Même le visible était à revoir. Les cartes étaient définitivement rebattues: il importait de le faire savoir. Autre grand chantier!

Cette décennie d'invention sera à écrire par d'autres, plus tard... Quand le temps et l'oubli auront cheminé sur l'action, poli l'instantané en hagiographie... Quand les mythes fondateurs auront épuisé, émoussé l'acuité des actes en «nostalgie de l'avenir».

L'aventure continue, ailleurs et autrement. On the road again!

\section{Les Nouvelles de l'Archéologie: le manifeste d'une discipline}

Anick Coudart

CNRS, Arizona State University

D e la gageure à la construction. Il y aura bientôt trente ans, un petit groupe de chercheurs s'était donné pour mission de construire l'archéologie métropolitaine française. ${ }^{\mathbf{1}}$ Lambition ne manquait pas d'immodestie, mais elle était enthousiaste, militante et totalement dénuée de carriérisme.

L'aventure commença au printemps 1978. À Paris, l'administrateur de la Fondation de la Maison des sciences de l'homme accueillait une table ronde sur les politiques de l'archéologie en Europe; il proposa à ses deux organisateurs (Chapelot, Schnapp 1984) de prendre en charge la fabrication d'un périodique consacré à l'archéologie, à l'image des petits bulletins scientifiques édités par la «Maison». Jean Chapelot souhaitait reprendre, en France, les thèmes de l'organisation britannique Rescue, fondée en 1971 par un petit groupe de pression ouvrant pour la protection, la conservation, l'enregistrement et l'interprétation des données archéologiques. Quelques jours plus tard, à Marseille, deux jeunes protohistoriens - Jean-Paul Demoule et l'auteur de ces lignes - rendaient visite au directeur du Laboratoire d'informatique pour les sciences de l'homme; ce dernier suggéra de créer une lettre d'information gratuite du type newsletter. Les quatre, rejoints par quelques autres, s'accordèrent: le bulletin serait scientifique, informatif et «militerait» pour le développement et la cohésion de l'archéologie. La Maison des sciences de l'homme releva le pari... Nouvelles de l'Archéologie était né [Fig.1].

C'est ainsi qu'en juillet 1979, un premier numéro $\left(\mathrm{n}^{\circ} \mathrm{O}\right)$ était distribué à 1100 archéologues et historiens. Jusqu'au numéro 10, la revue a été envoyée gratuitement aux 3000 personnes qui en avaient fait la demande. Qu'une revue aussi austère ait pu ensuite conquérir 1200 abonnés payants était assez exceptionnel. Qu'un éditeur privé (Errance) soit venu, neuf ans plus tard, solliciter la rédaction était une preuve du succès et du rôle que les Nouvelles avaient fini par jouer au sein de la communauté.

Pendant près de vingt-cinq ans, les éditoriaux et le contenu de la revue ont rendu compte 